\title{
Screening of differential microRNA expression in gastric signet ring cell carcinoma and gastric adenocarcinoma and target gene prediction
}

\author{
JIAN CHEN $^{1,2^{*}}$, DI SUN $^{1 *}$, HONGJIN CHU $^{1}$, ZHAOHUA GONG $^{2}$, CHENGLIN ZHANG $^{1}$, \\ BENJIAO GONG ${ }^{1}$, YAN LI ${ }^{1}$, NING LI $^{1}$ and LIXIN JIANG ${ }^{3}$ \\ ${ }^{1}$ Central Laboratory, ${ }^{2}$ Department of Oncology, and ${ }^{3}$ Department of Gastrointestinal Surgery, \\ Yantai Yuhuangding Hospital Affiliated to Qingdao University, Yantai, Shandong 264000, P.R. China
}

Received February 9, 2015; Accepted March 10, 2015

DOI: 10.3892/or.2015.3935

\begin{abstract}
Gastric signet ring cell carcinoma (GSRCC) is a unique pathological type of gastric carcinoma that is extremely invasive and has a poor prognosis after diagnosis. The expression of microRNAs has been closely linked to the carcinogenesis of gastric cancer and has been considered as a powerful prognostic marker. Distinctive expression of miRNAs in GSRCC was investigated in the present study. Samples of GSRCC were compared to that of intestinal gastric adenocarcinoma using Agilent microarray technique, and two differentially expressed miRNAs were identified, hsa-miR-665 and hsa-miR-95. qRT-PCR verification showed downregulation of both miRNAs in signet ring cell carcinoma and upregulation in gastric adenocarcinoma, which was not consistent with the results obtained by the microarray. Target gene prediction using online databases conferred two strong candidate genes, GLI2 and PLCG1. GO/KO analysis of these two genes showed close correlations with carcinogenesis and chemoresistance. It was concluded that hsa-miR-665 and hsa-miR-95 were downregulated in GSRCC but upregulated in intestinal gastric adenocarcinoma, and the relatively differential expression of the miRNAs negatively controlling their target genes could be closely related to the high invasive metastasis and chemoresistance of GSRCC.
\end{abstract}

\section{Introduction}

Gastric cancer is the second most common cancer in the Chinese population and the second leading cause of cancer-

Correspondence to: Dr Lixin Jiang, Department of Gastrointestinal Surgery, Yantai Yuhuangding Hospital Affiliated to Qingdao University, 20 East Yuhuangding Road, Yantai, Shandong 264000, P.R. China

E-mail: jianlixin1969@hotmail.com

"Contributed equally

Key words: microRNA, gastric signet ring cell carcinoma, gastric adenocarcinoma related death worldwide. More than $90 \%$ of all gastric cancer cases are gastric adenocarcinomas (GCs) (1), which are subdivided into intestinal- and diffuse-type according to the clinical and pathological characteristics (2). In recent years, the overall incidence of gastric cancer has decreased (3), yet the incidence of diffuse-type gastric cancer particularly that of the gastric signet ring cell carcinoma (GSRCC) has increased significantly, and tends to affect younger population (4). GSRCC is also called mucinous cell carcinoma, as cancerous cells are filled with large amounts of mucus and the nucleus is squeezed to one side and form a ring shape. Compared with the classic type of gastric cancer, GSRCC is more invasive and usually develops drug resistance; therefore, it is highly malignant and the prognosis is poor. Due to its complexity, current studies have mainly focused on the pathogenesis study of several disease-related genes (5-8).

microRNAs (miRNAs) are non-coding small singlestranded RNAs with a length of $20-25$ nucleotides, found in eukaryotes with functions of transcriptional and post-transcriptional regulation of gene expression. miRNA expression has been related to carcinogenesis and has been proposed as a potential biomarker in numerous studies (9-11). miRNAs promote mRNA degradation by inhibiting translation upon binding to the 3'-untranslated region (3'-UTR) of their target mRNA (12). Complementary binding of an miRNA and mRNA is not exclusive; a single miRNA can target hundreds of mRNAs, which was demonstrated in the present study and a single mRNA can be affected by multiple miRNAs (13-17). Consequently, miRNAs can act as tumor suppressors or possibly oncogenes depending on the target mRNAs $(18,19)$. Studies have shown that miRNAs have carcinogenic effects on GC and can be used as potential prognostic markers (20-23). Some studies show that a number of miRNAs are related to the growth and proliferation of GC (24-30), yet reports on GSRCC are rare.

We speculated that the invasiveness and resistance of GSRCC are likely due to the unique miRNA and mRNA regulation. In order to conduct a comparative analysis with intestinal-type gastric adenocarcinoma (IGA), we adopted microarray technology to identify the unique miRNAs and mRNAs that are related to the biological features of GSRCC. 
This should provide significant understanding of the mechanisms involved in GSRCC metastasis and chemotherapy resistance. Using the Agilent miRNA and mRNA microarray technology, miRNA/mRNA expression and identification in GSRCC and IGA were studied, respectively; bioinformatic analysis was utilized for the miRNA-mRNA integration study. Differentially expressed miRNAs in GSRCC were thoroughly studied to determine the biological function of the targeted mRNAs in the occurrence of cancer. miRNAs regulate genes that act as prognostic factors for patients with GC (12). The present study aimed to compare the miRNA profile of IGA and GSRCC tissues and their targeted mRNAs.

\section{Materials and methods}

Sample preparation. Fifty-two human gastric tissue specimens were collected from the Yantai Yuhuangding Hospital specimen bank from 26 subjects diagnosed with gastric cancer. The specimens included 13 GSRCC specimens (T1), 13 GSRCC paracancerous tissues (P1) from the same group of subjects, 13 IGA samples (T2) and 13 IGA paracancerous tissues (P2). All samples were collected and kept in liquid nitrogen within $30 \mathrm{~min}$ after the tissue was assembled and then transferred for storage at $-80^{\circ} \mathrm{C}$. All subjects were fully informed and signed consent forms. The present study was approved by the Human Research Ethics Committee of Yantai Yuhuangding Hospital. Four samples were selected from each group for Agilent miRNA and mRNA chip analysis.

Total RNA extraction. According to the manufacturer's instructions (TRIzol; Invitrogen, Gaithersburg, MD, USA) total RNA was extracted from 52 gastric cancer tissue samples individually. Samples were then chosen for RNA column purification using NucleoSpin ${ }^{\circledR}$ RNA Clean-up kit (Macherey-Nagel, Germany). Samples were quantified with a spectrophotometer, and tested with formaldehyde denaturing gel electrophoresis for quality inspection. Four pairs of samples were randomly chosen from the GSRCC and IGA groups, and prepared for cDNA synthesis using the EasyScript First-Strand cDNA Synthesis SuperMix kit (Beijing Gold-Tide Biotechnology Co., Ltd.).

Agilent miRNA and mRNA chip (completed by Beijing Boao Biochip Co., Ltd., Beijing Biochip National Engineering Center). The miRNA and mRNA synthetic products were fluorescently-labeled and dissolved in hybridization solution. After repeated washing and drying, the Agilent chips (Agilent Technologies, Santa Clara, CA, USA) were scanned with Agilent chip scanner (G2565CA), and hybridization images were obtained. The images were analyzed and transferred into digital signals using the Agilent Feature Extraction (v10.7) software. GeneSpring GX software (Agilent Technologies) was then used for normalization using the percentile shift method. The differences between the four groups were analyzed using GeneSpring software. A p $<0.05$ and absolute fold-change $\geq 2$, and the flag marked as detected were used as standards for differences in miRNA and mRNA screening.

Quantitative RT-PCR verification of miRNA and $m R N A$ expression in GSRCC. All extracted samples from the GSRCC and IGA groups were subjected to qRT-PCR. The Platinum ${ }^{\circledR}$ RTS SYBR ${ }^{\circledR}$-Green qPCR SuperMix-UDG kit (Invitrogen) was used for miRNA and mRNA differential expression qRT-PCR verification with a reaction system of $20 \mu \mathrm{l}$, and each miRNA and mRNA sample was provided with 3-wells and repeated 3 times. Primer designs are listed in Table I. U6 and GAPDH correction was used for targeted miRNA and mRNA expression, respectively. Analysis was carried out using Rotor-Gene Q Series software. $\Delta \mathrm{Ct}$ was calculated using the equation $\Delta \mathrm{Ct}=\mathrm{Ct}_{\text {miRNA }}-\mathrm{Ct}_{\text {miR-U6 }}$; the relative miRNA expression was calculated using the $2^{-\Delta \Delta \mathrm{Ct}}$ method.

Bioinformatic analysis of the targeted miRNAs and GO/KO analysis. The differentially expressed miRNAs were analyzed and predicted with 10 online gene databases, including DIANAmT, miRanda, miRDB, miRWalk, RNAhybrid, PICTAR4, PICTAR5, PITA, RNA22 and Targetscan (the differentially expressed miRNAs which were successfully assigned to target genes by more than two database were selected). Due to the fact that miRNAs negatively regulate target genes, miRNAs and mRNAs with differential expression were divided into groups of upregulation and downregulation, and applied for $\mathrm{GO} / \mathrm{KO}$ analysis $(\mathrm{p}<0.05$ as the screening filter). The selected gene was matched with those detected by microarray, and a final target gene of GSRCC that was regulated by miRNA was confirmed and further studied.

Statistical analysis. Statistical analysis was performed using SPSS 17.0 software. All data were shown as mean \pm SEM. The data were analyzed for statistical significance using an unpaired Student's t-test. For all the experiments, any p-value $<0.05$ was considered to indicate a statistically significant result.

\section{Results}

Differentially expressed miRNAs were identified from the GSRCC (T1/P1) and IGA (T2/P2) groups using the Agilent mRNA chip. Fifteen differentially expressed miRNAs were recognized in the T1/P1 group (four pairs of sample) $(\mathrm{p}<0.05)$; 13 were upregulated and 2 were downregulated (Table II). In the T2/P2 group, 39 miRNAs were upregulated whereas 6 were downregulated $(\mathrm{p}<0.05)$ (Table III). By comparing the two groups, 10 commonly expressed miRNAs were prominent, including 9 that were upregulated and 1 that was downregulated.

Differentially expressed $m R N A s$ were identified from the GSRCC (T1/P1) and IGA (T2/P2) groups. A total number of 760 differentially expressed genes were identified between $\mathrm{T} 1$ and P1, including 364 upregulated genes and 396 downregulated genes. Differentially expressed genes $(1,745)$ were identified between T2 and P2, including 855 upregulated and 890 downregulated genes. Overall, 581 genes were mutually expressed between the two comparative groups, with 288 upregulated and 293 downregulated genes (Fig. 1).

Target gene prediction of differentially expressed miRNAs in $T 1 / P 1$. Target gene prediction was carried out by comparison of the binding site of the $10 \mathrm{~T} 1 / \mathrm{P} 1$ specifically expressed 
Table I. Primer design.

\begin{tabular}{lll}
\hline Gene name & & \multicolumn{1}{c}{ Primer sequence } \\
\hline hsa-miR-665 & Forward primer & 5'-GCGGCGGACCAGGAGGCTGAGG-3' \\
& RT primer & 5'-GTCGTATCCAGTGCAGGGTCCGAGGTATTCGCACTGGATACGACAGGGGC-3' \\
& Reverse primer & 5'-ATCCAGTGCAGGGTCCGAGG-3' \\
hsa-miR-95-3p & Forward primer & 5'-GCGGCGGTTCAACGGGTATTTA-3' \\
& RT primer & 5'-GTCGTATCCAGTGCAGGGTCCGAGGTATTCGCACTGGATACGACTGCTCA-3' \\
& Reverse primer & 5'-ATCCAGTGCAGGGTCCGAGG-3' \\
U6 & Forward primer & 5'-CTCGCTTCGGCAGCACA-3' \\
& RT primer & 5'-AACGCTTCACGAATTTGCGT-3' \\
GLI2 & Forward primer & 5'-GTAAGCAGGAGGCTGAGGTG-3' \\
& Reverse primer & 5'-GCTCGTTGTTGATGTGATGC-3' \\
PLCG1 & Forward primer & 5'-AGAACATGCTGTCCCAGGTC-3' \\
& Reverse primer & 5'-AACTGCCCGTAGGTGATGTC-3' \\
GAPDH & Forward primer & 5'-GACCACAGTCCATGCCATCACT-3' \\
& Reverse primer & 5'-TCCACCACCCTGTTGCTGTA-3' \\
\hline
\end{tabular}

Table II. Expression of miRNAs in GSRCC (T1) and paracancerous tissues $(\mathrm{P} 1)$.

\begin{tabular}{|c|c|c|c|}
\hline $\begin{array}{l}\text { Upregulated } \\
\text { miRNAs }\end{array}$ & $\begin{array}{l}\text { Fold- } \\
\text { change }\end{array}$ & $\begin{array}{c}\text { Downregulated } \\
\text { miRNAs }\end{array}$ & $\begin{array}{l}\text { Fold- } \\
\text { change }\end{array}$ \\
\hline hsa-miR-181a-2-3p & 29.86 & hsa-miR-148a-5p & 3.65 \\
\hline hsa-miR-154-3p & 27.05 & hsa-miR-148a-3p & 3.04 \\
\hline hsa-miR-550a-3p & 24.92 & & \\
\hline hsa-miR-214-5p & 19.55 & & \\
\hline hsa-miR-10a-3p & 17.78 & & \\
\hline hsa-miR-3605-5p & 16.21 & & \\
\hline hsa-miR-6073 & 15.49 & & \\
\hline hsa-miR-224-3p & 14.90 & & \\
\hline hsa-miR-4260 & 14.26 & & \\
\hline hsa-miR-584-5p & 14.07 & & \\
\hline hsa-miR-665 & 6.81 & & \\
\hline hsa-miR-93-3p & 5.76 & & \\
\hline hsa-miR-95a & 2.07 & & \\
\hline
\end{tabular}

Fold-change represents the amount of miRNA expression in $\mathrm{T} 1$ compared to that in P1; miRNAs highlighted in bold are the ones commonly expressed in both GSRCC and IGA groups. "Represents miRNAs predicted with target genes. GSRCC, gastric signet ring cell carcinoma; IGA, intestinal gastric adenocarcinoma.

miRNAs (Table II) to a series number of gene sequences in the databases. hsa-miR-665 was matched to 218 potential target genes, among them 134 were upregulated genes and 84 were downregulated; hsa-miR-95 was matched to a total number of 67 target genes with 47 being upregulated and 20 being downregulated.

qRT-PCR verification of hsa-miR-665 and hsa-miR-95 in the GSRCC tissue. qRT-PCR showed that the expression

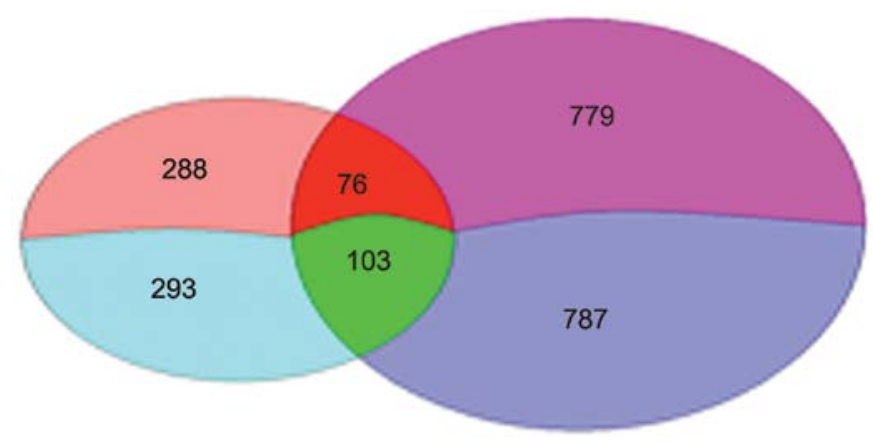

Figure 1. Venn diagram showing gene expression in the T1/P1 and T2/P2 groups. The top section of the left Venn diagram (orange and red) represents 364 upregulated genes in the T1/P1 groups, whereas the bottom (blue and green) represents 396 downregulated genes in the T1/P1 groups; the top section of the right Venn diagram (pink and red) represents 855 upregulated genes in T2/P2, whereas the bottom (purple and green) represents 890 downregulated genes in $\mathrm{T} 2 / \mathrm{P} 2$; the overlapping red region represents 76 genes that are commonly upregulated in both groups whereas green represents 103 genes commonly downregulated in both groups.

of hsa-miR-665 and hsa-miR-95 was relatively reduced by $0.30 \pm 0.04$ - and $0.36 \pm 0.05$-fold in the $\mathrm{T} 1 / \mathrm{P} 1$ groups. In the T2/P2 groups hsa-miR-665 and hsa-miR-95 were relatively increased by $1.60 \pm 0.01$ - and $1.49 \pm 0.08$-fold (Fig. 2).

Target gene screening of miRNAs from T1/P1 and $G O / K O$. Twenty-eight upregulated target genes were selected for hsa-miR-665 from T1/P1; 7 were also upregulated in hsa-miR-95 (Table IV). Comprehensive networking of the functional features related to carcinogenesis were produced and the genes with the highest interest in the present study, GLI2 and PLCG1, were selected and GO and KO plots were constructed (Fig. 4). GO analysis showed that the functions of the GLI2 gene related to tumors mainly include: transcription factor activation, ion binding, regulation of RNA polymerase II promoter, axon guidance, cell proliferation 
Table III. Expression of miRNAs in classic gastric adenocarcinoma tissue (T2) and its paracancerous tissue (P2).

\begin{tabular}{|c|c|c|c|c|c|}
\hline Upregulated miRNAs & Fold-change & Upregulated miRNAs & Fold-change & Downregulated miRNAs & Fold-change \\
\hline hsa-miR-196a-5p & 133.31 & hsa-miR-224-3p & 13.38 & hsa-miR-375 & 3.20 \\
\hline hsa-miR-499a-5p & 94.57 & hsa-miR-378b & 13.38 & hsa-miR-31-5p & 3.00 \\
\hline hsa-miR-196b-5p & 66.42 & hsa-miR-378b & 12.25 & hsa-miR-148a-3p & 2.62 \\
\hline hsa-miR-1180 & 41.50 & hsa-miR-4685-5p & 10.90 & hsa-miR-551b-3p & 2.40 \\
\hline hsa-miR-1290 & 38.29 & hsa-miR-19b-1-5p & 10.07 & hsa-miR-4687-3p & 2.35 \\
\hline hsa-miR-550a-3-5p & 34.48 & hsa-miR-301b & 9.98 & hsa-miR-572 & 2.04 \\
\hline hsa-miR-592 & 30.09 & hsa-miR-149-3p & 6.45 & & \\
\hline hsa-miR-4535 & 27.54 & hsa-miR-335-5p & 2.75 & & \\
\hline hsa-miR-584-5p & 26.68 & hsa-miR-93-5p & 2.54 & & \\
\hline hsa-miR-4710 & 25.39 & hsa-miR-301a-3p & 2.40 & & \\
\hline hsa-miR-4776-5p & 22.52 & hsa-miR-361-5p & 2.35 & & \\
\hline hsa-miR-550a-3p & 22.35 & hsa-miR-181d & 2.34 & & \\
\hline hsa-miR-4419b & 20.33 & hsa-miR-221-3p & 2.23 & & \\
\hline hsa-miR-374b-3p & 19.68 & hsa-miR-181c-5p & 2.18 & & \\
\hline hsa-miR-652-5p & 17.84 & hsa-miR-21-5p & 2.13 & & \\
\hline hsa-miR-670 & 17.78 & hsa-miR-19a-3p & 2.09 & & \\
\hline hsa-miR-10a-3p & 15.27 & hsa-miR-10a-5p & 2.07 & & \\
\hline hsa-miR-3154 & 14.98 & hsa-miR-622 & 2.02 & & \\
\hline hsa-miR-1273g-5p & 14.13 & hsa-miR-3911 & 2.01 & & \\
\hline hsa-miR-5196-5p & 13.96 & & & & \\
\hline
\end{tabular}

Fold-change represents the amount of miRNA expression in T2 compares to that in P2. miRNAs highlighted in bold are the ones commonly expressed in both the GSRCC and IGA groups. GSRCC, gastric signet ring cell carcinoma; IGA, intestinal gastric adenocarcinoma.

and DNA replication. KO analysis showed that PLCG1 was mainly involved in the signaling pathway of tumors including the phosphoinositide, ErbB and calcium signaling pathways, VEGF pathway, and Fce RI signaling pathways. As for hsa-mir-95, there were only two genes, TNFRSF19 and RAB11FIP4, predicted with the criteria, and only RAB11FIP4 was predicted as a target gene by over four databases, and both were comparatively irrelevant. Therefore, they were not further validated.

qRT-PCR verification of GLI2 and PLCG1. GLI2 and PLCG1 were upregulated in the T1/P1 group by $7.79 \pm 0.31$ - and $5.09 \pm 0.03$-fold, relatively. In the $\mathrm{T} 2 / \mathrm{P} 2$ groups, both were downregulated by $0.18 \pm 0.42$ - and $0.38 \pm 0.03$-fold, respectively (Fig. 3).

\section{Discussion}

GSRCC is a unique pathological type of gastric carcinoma. The IGA related overexpression of EGF and mutation of the anticancer gene P53 have also been detected in GSRCC, suggesting a common pathogenesis $(31,32)$. However, as one of the most highly malignant tumors, GSRCC also presents powerful features of invasion, metastasis and chemoresistance. At the early stage, the tumor often fails to present typical clinical manifestations, but progresses rapidly with metastasis. Therefore, the majority of patients are diagnosed with advanced stage and lose the chance for surgical treatment. Patients with GSRCC are relatively insensitive to chemotherapy and radio-
Table IV. Target gene prediction of hsa-miR-665 in the GSRCC tissue.

hsa-miR-665 upregulation (no.)

\begin{tabular}{llll}
\hline PPAPDC1A (4) & CHSY3 (3) & CHN1 (2) & VWA1 (3) \\
LPP (2) & FSTL1 (5) & SEMA5B (2) & ODZ4 (2) \\
CRABP2 (2) & AQP1 (2) & SEMA6B (2) & GLI2 (5) \\
AQP1 (2) & APLN (3) & LINGO1 (5) & FSTL3 (2) \\
TRPM5 (4) & SGCD (4) & PXDN (3) & NUMBL (2) \\
MRAS (2) & IGFBP4 (4) & NUMBL (2) & CYP26B1 (5) \\
CHIT1 (5) & PTGIR (3) & CLCF1 (3) & SIPA1L2 (5) \\
DOK5 (2) & PLCG1 (4) & & \\
\hline
\end{tabular}

${ }^{a}$ Indicates genes that were highlighted in GO and $\mathrm{KO}$ analysis. Genes in bold were also upregulated in hsa-miR-95.

therapy; hence, poor prognosis becomes a huge hurdle in the clinical treatment for this type of tumor. Previous studies have shown that increased expression of PKM2 and E-cadherin, and decreased expression of TMEM207 in GSRCC are closely related to its characteristics of invasion and metastasis (33-35). Another study also showed that inherent chemoresistance of GSRCC and a delay in definitive surgery may favor tumor progression (36). Although these studies indicate a direction for GSRCC research, the occurrence of GSRCC involves a variety of abnormally expressed tumor-related genes, in a 


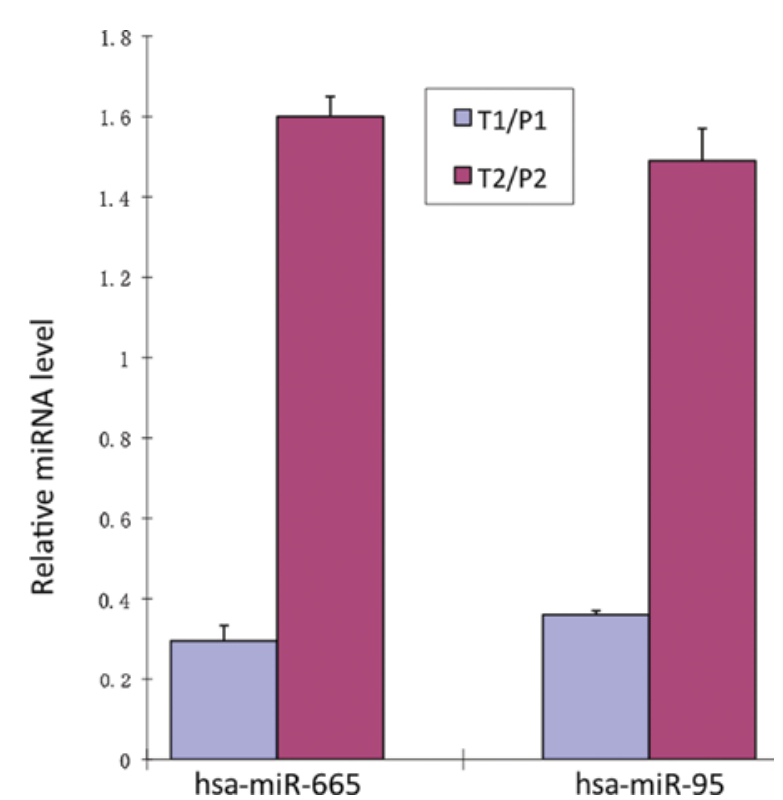

Figure 2. A bar chart showing the expression levels of hsa-miR-665 and hsa-miR-95 in the T1/P1 and T2/P2 groups in the qRT-PCR verification. Error bars represent the average expression of the four samples.

multi-stage process. Therefore, it is imperative to screen for novel biomarkers that are differentially expressed in GSRCC, and investigate drugs that target chemoresistance in GSRCC. With a very limited number of studies in this area, the present study aimed to identify specifically expressed biomarkers between GSRCC and IGA, and identify unique biological features of this malignancy.

Some recent studies have demonstrated that miRNA expression is linked to carcinogenesis by regulating its target genes. These miRNAs also play a part as tumor-suppressor genes, such as miRNA-370, miRNA-520d-3p and miR181a-5p (37-39); or as oncogenes, such as miRNA-21, miR-18a and miR-544a (40-42). We randomly selected 4 pairs of GSRCC samples and 4 pairs of IGA samples, and conducted a comparative analysis of the differentially expressed miRNAs using microarray analysis. As a result, two miRNAs, hsa-miR665 and hsa-miR-95, were selected for further verification.

hsa-miR-665 is located at $14 \mathrm{q} 32.2$, with a length of 20 amino acids. Its expression has been reported to inhibit the expression of B7-H3 in breast cancer (17), yet its mechanism remains unknown. In the present study, qRT-PCR results showed that hsa-miR-665 was downregulated in GSRCC, whereas microarray analysis showed contradictory results. Repeated qRT-PCR verification of hsa-miR-665 also showed a decreased expression of hsa-miR-665, which is consistent with our previous results and that reported in breast cancer. Thus, it was speculated that hsa-miR-665 may play a role as a tumor-suppressor gene. In order to predict its mechanism of action, a number of potential target genes were evaluated. Some studies have reported upregulation of hsa-miR-665 expression in a series of cancers, for example in esophageal squamous cell carcinoma (43) and bladder urothelial carcinoma (BUC) (44). hsa-miR-95 is located at 4p16.1, with a length of 22 amino acids. hsa-miR-95 plays a role as a protooncogene in the occurrence and development of a number of tumors, such as colon, cervical and pancreatic cancer, in which

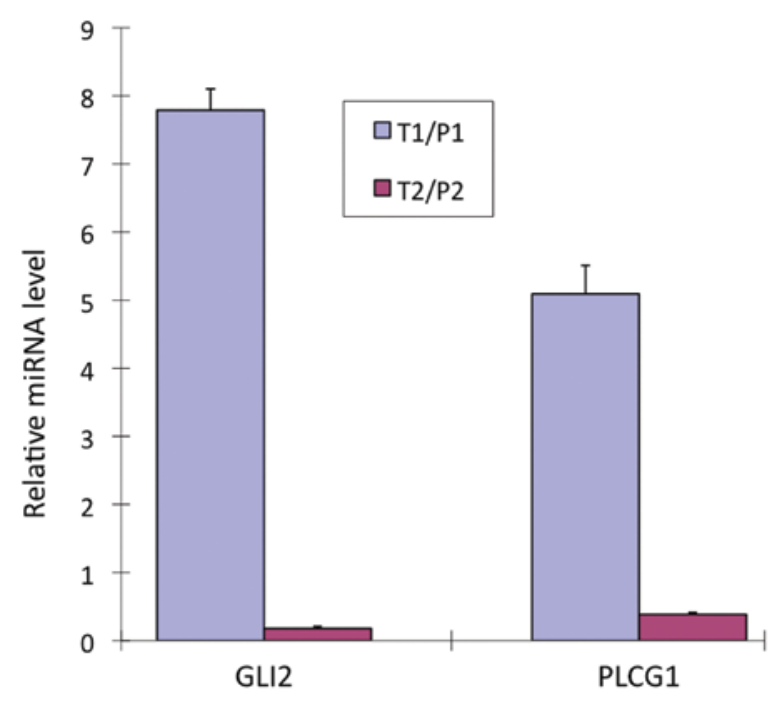

Figure 3. A bar chart showing the expression levels of GLI2 and PLCG1 in the T1/P1 and T2/P2 groups in qRT-PCR verification. Error bars represent average expression of four samples.

upregulation of hsa-miR-95 was observed (45-47). Recent studies found that hsa-miR-95 is a target for the treatment of liver cancer with Brucein D, and it is also closely related to radiotherapy and chemotherapy tolerance (48-50). In addition, downregulation of hsa-miR-95 expression was detected in human glioblastoma (51). Therefore, hsa-miR-95 may have different functions in different types of tumors. qRT-PCR verification showed downregulation of hsa-miR-665 and hsa-miR-95 in the GSRCC samples, and upregulation in IGA samples, which was different from the results obtained by the microarray analysis. Repeated qRT-PCR showed a similar pattern of expression, which may be explained by different functional features of these two miRNAs in different types of gastric cancer. In order to establish possible functions of hsa-miR-665 and hsa-miR-95 in GSRCC, target genes were predicted by filtering potential genes from a number of databases and $\mathrm{GO} / \mathrm{KO}$ analysis. Preliminary results revealed two genes, GLI2 and PLCG1, with the greatest connections in terms of tumorigenesis.

GLI2 is located in $2 \mathrm{q} 14$, a member of the zinc finger transcription factor GLI family. Studies found that there was increased GLI2 activity in gastric cancer, lung squamous cell, pancreatic and basal cell carcinoma, and melanoma and other tumors, possibly by the activation of Hedgehog signaling pathway to promote tumor invasion and induce chemoresistance (52-56). PLCG1, also called PLC $\gamma 1$, is located in 20q12-q13.1. PLC- $\gamma 1$ is a subtype of phospholipase (PLC). Many cytokines reply on PLC to regulate cell metabolism. Studies have found that PLC- $\gamma 1$ was upregulated in breast and oral cancer and other tumors $(57,58)$, and mutations in PLC- $\gamma 1$ are associated with the incidence of many types of cancers. It also promotes tumor growth and migration (58-62). The activation of PLC- $\gamma 1$ promotes tumor cell metastasis by triggering a series of signaling pathways including the PKC signaling pathway, calcium signaling pathway, Ras/ERK, VEGF signaling and MAPK pathways; it also promotes upregulation of the MDR1 gene and induces resistance of tumor cells to chemotherapeutic drugs (63-67). 


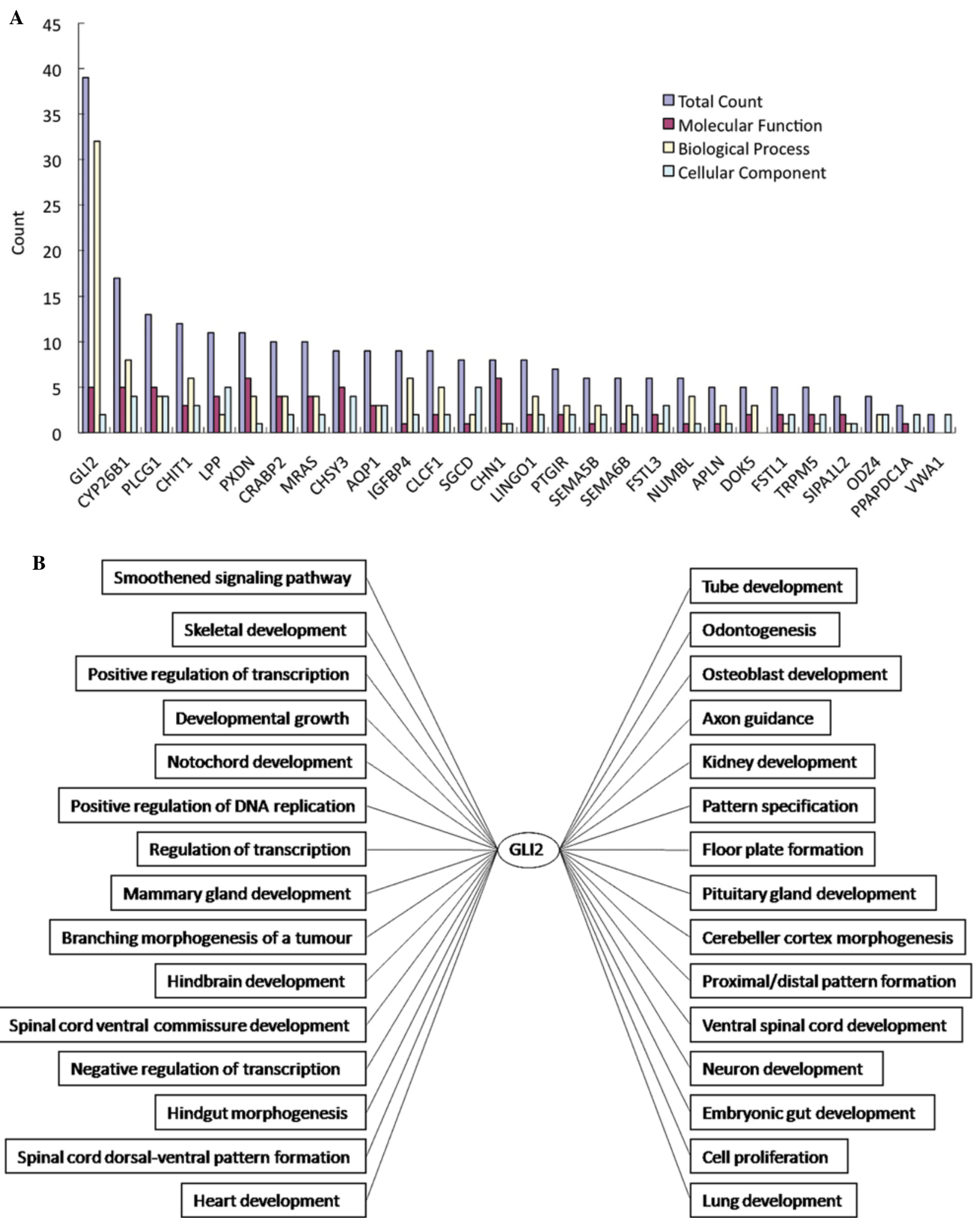

Figure 4. Go and KO network of genes predicted for hsa-miR-665 using CapitalBio Molecule Annotation System (MAS). (A) A bar chart shows that the variety of functions predicted for the targeted genes are mainly related to three main functions including molecular function, biological process and cellular component. The x-axis represents 28 genes and the y-axis represents the number of functions. (B) The GO functional network analysis of GLI2, when the 28 targeted genes were opt for $\mathrm{GO}$ and $\mathrm{KO}$ analysis.

qRT-PCR verification of GLI2 and PLCG1 showed upregulation in GSRCC but downregulation in IGA samples. The relative expression of GLI2 was inversely regulated by hsa-miR-665 and hsa-miR-95; PLCG1 expression was inversely regulated by hsa-miR-665. Together with the results of the $\mathrm{GO} / \mathrm{KO}$ analysis, it was speculated that hsa-miR-665 and hsa-miR-95 promote metastasis and chemoresistance in GSRCC by upregulating its target genes, in this case GLI2 


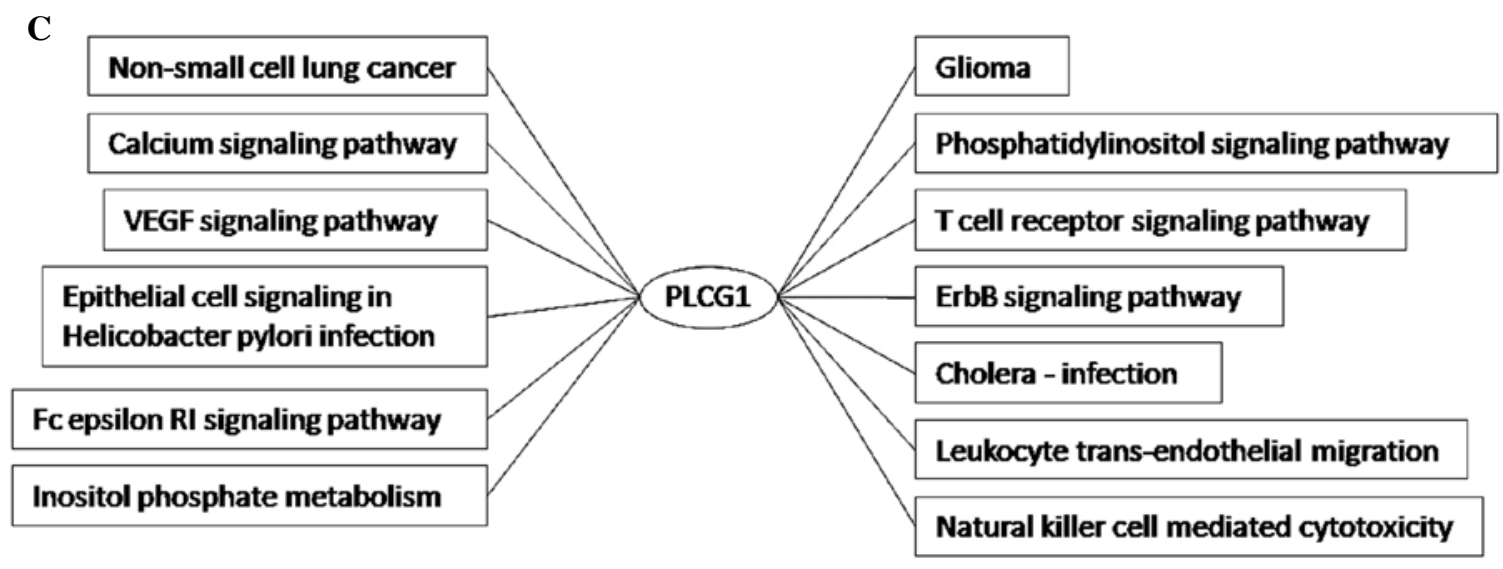

Figure 4. Continued. (C) KO pathway networking centered by PLCG1.

and PLCG1. And the distinct expression of these miRNAs could be the key to the different molecular pathogenesis of GSRCC and IGA. Moreover, it was observed that the results of the qRT-PCR verification was not consistent with that of the microarray analysis. This could be due to relatively a high false-positive rate of the array analysis, or the 4 pairs of samples chosen from each GC type did not represent all samples in this study.

In addition, miRNA verification showed differential expression of hsa-miR-665 and hsa-miR-95 in both the GSRCC and intestinal IGA, yet the difference was more apparent in GSRCC. Retrospective analysis of the miRNA results of the target genes showed that genes differentially expressed in GSRCC were also present in the intestinal type, which is distinctive from the results from the array analysis. This is possibly due to different sensitivity between the detection methods.

In summary, invasion and metastasis are the main characteristics of GSRCC, which also explains its poor prognosis and high mortality rate. Chemoresistance is a major hurdle in clinical treatment of most cancers. For the first time, the present study showed that hsa-miR-665 and hsa-miR-95 are downregualted in the GSRCC type of gastric cancer, but upregulated in IGA, and the target genes present with the opposite pattern of expression. Therefore, the relatively differential expression of miRNAs negatively controlling their target genes could be closely related to the high invasive metastasis and chemoresistance of GSRCC. The mechanism of its molecular pathogenesis requires further study. Using microarray technology, the present study identified for the first time that hsa-miR-665 and hsa-miR-95 exhibit a significant differential expression pattern in GSRCC compare to intestinal IGA. Target gene screening by bioinformatic analysis of corresponding miRNAs showed that differentially expressed miRNAs and mRNAs are likely to have a significant correlation with invasion and metastasis and multidrug resistance of gastric signet ring cell, but its mechanism of action remains to be further evaluated. Overall, these two markers identified in the present study should provide information concerning the unique biological characteristics of GSRCC, which could provide the theoretical basis for therapeutic development for this type of cancer.

\section{Acknowledgements}

This study was supported by the National Natural Science Foundation of China (no. 81071758), Shandong Province Young and Middle-Aged Scientists Research Awards Fund (BS2009SW052, 2008BS02012), and the Yantai Science and Technology Program (2012085).

\section{References}

1. Piazuelo MB and Correa P: Gastric cáncer: Overview. Colomb Med 44: 192-201, 2013.

2. Lauren P: The two histological main types of gastric carcinoma: Diffuse and so-called intestinal-type carcinoma. An attempt at a histo-clinical classification. Acta Pathol Microbiol Scand 64: 31-49, 1965.

3. Siegel R, Naishadham D and Jemal A: Cancer statistics, 2013. CA Cancer J Clin 63: 11-30, 2013.

4. Kim BS, Oh ST, Yook JH and Kim BS: Signet ring cell type and other histologic types: Differing clinical course and prognosis in T1 gastric cancer. Surgery 155: 1030-1035, 2014.

5. Kwon KJ, Shim KN, Song EM, Choi JY, Kim SE, Jung HK and Jung SA: Clinicopathological characteristics and prognosis of signet ring cell carcinoma of the stomach. Gastric Cancer 17: 43-53, 2014.

6. Tabouret T, Dhooge M, Rouquette A, Brezault C, Beuvon F, Chaussade $\mathrm{S}$ and Coriat R: Gastric signet ring cell adenocarcinoma: A distinct entity. Presse Med 43: 353-357, 2014 (In French).

7. Huh CW, Jung H, Kim JH, Lee YC, Kim H, Kim H, Yoon SO, Youn YH, Park H, Lee SI, et al: Signet ring cell mixed histology may show more aggressive behavior than other histologies in early gastric cancer. J Surg Oncol 107: 124-129, 2013.

8. Hass HG, Smith U, Jäger C, Schäffer M, Wellhäuber U, Hehr T, Markmann HU, Nehls O and Denzlinger C: Signet ring cell carcinoma of the stomach is significantly associated with poor prognosis and diffuse gastric cancer (Lauren's): Single-center experience of 160 cases. Onkologie 34: 682-686, 2011.

9. Llauradó M, Majem B, Altadill T, Lanau L, Castellví J, Sánchez-Iglesias JL, Cabrera S, De la Torre J, Díaz-Feijoo B, Pérez-Benavente A, et al: MicroRNAs as prognostic markers in ovarian cancer. Mol Cell Endocrinol 390: 73-84, 2014.

10. Mulrane L, Klinger R, McGee SF, Gallagher WM and O'Connor DP: microRNAs: A new class of breast cancer biomarkers. Expert Rev Mol Diagn 14: 347-363, 2014.

11. Xue Y, Abou Tayoun AN, Abo KM, Pipas JM, Gordon SR, Gardner TB, Barth RJ Jr, Suriawinata AA and Tsongalis GJ: MicroRNAs as diagnostic markers for pancreatic ductal adenocarcinoma and its precursor, pancreatic intraepithelial neoplasm. Cancer Genet 206: 217-221, 2013.

12. Valencia-Sanchez MA, Liu J, Hannon GJ and Parker R: Control of translation and mRNA degradation by miRNAs and siRNAs. Genes Dev 20: 515-524, 2006. 
13. Selbach M, Schwanhäusser B, Thierfelder N, Fang Z, Khanin R and Rajewsky N: Widespread changes in protein synthesis induced by microRNAs. Nature 455: 58-63, 2008.

14. Li H, Meng F, Ma J, Yu Y, Hua X, Qin J and Li Y: Insulin receptor substrate-1 and Golgi phosphoprotein 3 are downstream targets of miR-126 in esophageal squamous cell carcinoma. Oncol Rep 32: 1225-1233, 2014.

15. Konno Y, Dong P, Xiong Y, Suzuki F, Lu J, Cai M, Watari H, Mitamura T, Hosaka M, Hanley SJ, et al: MicroRNA-101 targets EZH2, MCL-1 and FOS to suppress proliferation, invasion and stem cell-like phenotype of aggressive endometrial cancer cells. Oncotarget 5: 6049-6062, 2014.

16. Zhong D, Huang G, Zhang Y, Zeng Y, Xu Z, Zhao Y, He X and He F: MicroRNA-1 and microRNA-206 suppress LXR $\alpha$-induced lipogenesis in hepatocytes. Cell Signal 25: 1429-1437, 2013.

17. Nygren MK, Tekle C, Ingebrigtsen VA, Mäkelä R, Krohn M, Aure MR, Nunes-Xavier CE, Perälä M, Tramm T, Alsner J, et al: Identifying microRNAs regulating B7-H3 in breast cancer: The clinical impact of microRNA-29c. Br J Cancer 110: 2072-2080, 2014.

18. Garzon R, Heaphy CE, Havelange V, Fabbri M, Volinia S, Tsao T, Zanesi N, Kornblau SM, Marcucci G, Calin GA, et al: MicroRNA 29b functions in acute myeloid leukemia. Blood 114 5331-5341, 2009.

19. Shenouda SK and Alahari SK: MicroRNA function in cancer: Oncogene or a tumor suppressor? Cancer Metastasis Rev 28: 369-378, 2009.

20. Ishiguro $\mathrm{H}$, Kimura $\mathrm{M}$ and Takeyama $\mathrm{H}$ : Role of microRNAs in gastric cancer. World J Gastroenterol 20: 5694-5699, 2014.

21. Wang M, Zhao C, Shi H, Zhang B, Zhang L, Zhang X, Wang S, Wu X, Yang T, Huang F, et al: Deregulated microRNAs in gastric cancer tissue-derived mesenchymal stem cells: Novel biomarkers and a mechanism for gastric cancer. Br J Cancer 110: 1199-1210, 2014.

22. Brenner B, Hoshen MB, Purim O, David MB, Ashkenazi K, Marshak G, Kundel Y, Brenner R, Morgenstern S, Halpern M, et al: MicroRNAs as a potential prognostic factor in gastric cancer. World J Gastroenterol 17: 3976-3985, 2011.

23. Lim JY, Yoon SO, Seol SY, Hong SW, Kim JW, Choi SH, Lee JS and Cho JY: Overexpression of miR-196b and HOXA10 characterize a poor-prognosis gastric cancer subtype. World J Gastroenterol 19: 7078-7088, 2013.

24. Peng Y, Liu YM, Li LC, Wang LL and Wu XL: microRNA-503 inhibits gastric cancer cell growth and epithelial-to-mesenchymal transition. Oncol Lett 7: 1233-1238, 2014.

25. Shen ZY, Zhang ZZ, Liu H, Zhao EH and Cao H: miR-375 inhibits the proliferation of gastric cancer cells by repressing ERBB2 expression. Exp Ther Med 7: 1757-1761, 2014.

26. Ren J, Huang HJ, Gong Y, Yue S, Tang LM and Cheng SY: MicroRNA-206 suppresses gastric cancer cell growth and metastasis. Cell Biosci 4: 26, 2014.

27. Peng Y, Guo JJ, Liu YM and Wu XL: MicroRNA-34A inhibits the growth, invasion and metastasis of gastric cancer by targeting PDGFR and MET expression. Biosci Rep 34: 34, 2014.

28. Tsai MM, Wang CS, Tsai CY, Chen CY, Chi HC, Tseng YH, Chung PJ, Lin YH, Chung IH, Chen CY, et al: MicroRNA196a/-196b promote cell metastasis via negative regulation of radixin in human gastric cancer. Cancer Lett 351: 222-231, 2014.

29. Wang T, Ge G, Ding Y, Zhou X, Huang Z, Zhu W, Shu Y and Liu P: MiR-503 regulates cisplatin resistance of human gastric cancer cell lines by targeting IGF1R and BCL2. Chin Med J 127: 2357-2362, 2014

30. Yang M, Shan X, Zhou X, Qiu T, Zhu W, Ding Y, Shu Y and Liu P: miR-1271 regulates cisplatin resistance of human gastric cancer cell lines by targeting IGF1R, IRS1, mTOR, and BCL2. Anticancer Agents Med Chem 14: 884-891, 2014.

31. Aizawa K, Muto I, Suzuki S, Tanaka N, Yabusaki H, Tanaka S, Katayanagi N, Suzuki T, Tanaka O and Muto T: Augmentation of 5-fluorouracil cytotoxicity by epidermal growth factor in a newly established human signet-ring cell carcinoma of the stomach in culture. Surg Today 24: 420-428, 1994.

32. Shimada S, Mimata A, Sekine M, Mogushi K, Akiyama Y, Fukamachi H, Jonkers J, Tanaka H, Eishi Y and Yuasa Y: Synergistic tumour suppressor activity of E-cadherin and p53 in a conditional mouse model for metastatic diffuse-type gastric cancer. Gut 61: 344-353, 2012.

33. Takeuchi T, Adachi Y and Nagayama T: A WWOX-binding molecule, transmembrane protein 207 , is related to the invasiveness of gastric signet-ring cell carcinoma. Carcinogenesis 33: $548-554,2012$
34. Humar B, Blair V, Charlton A, More H, Martin I and Guilford P: E-cadherin deficiency initiates gastric signet-ring cell carcinoma in mice and man. Cancer Res 69: 2050-2056, 2009.

35. Lim JY, Yoon SO, Seol SY, Hong SW, Kim JW, Choi SH and Cho JY: Overexpression of the M2 isoform of pyruvate kinase is an adverse prognostic factor for signet ring cell gastric cancer. World J Gastroenterol 18: 4037-4043, 2012.

36. Piessen G, Messager M, Le Malicot K, Robb WB, Di Fiore F, Guilbert M, Moreau M, Christophe V, Adenis A and Mariette C: Phase II/III multicentre randomised controlled trial evaluating a strategy of primary surgery and adjuvant chemotherapy versus peri-operative chemotherapy for resectable gastric signet ring cell adenocarcinomas - PRODIGE 19 - FFCD1103 - ADCI002. BMC Cancer 13: 281, 2013.

37. Chen XP, Chen YG, Lan JY and Shen ZJ: MicroRNA-370 suppresses proliferation and promotes endometrioid ovarian cancer chemosensitivity to cDDP by negatively regulating ENG. Cancer Lett 353: 201-210, 2014.

38. Li R, Yuan W, Mei W, Yang K and Chen Z: MicroRNA 520d-3p inhibits gastric cancer cell proliferation, migration, and invasion by downregulating EphA2 expression. Mol Cell Biochem 396: 295-305, 2014.

39. Korhan P, Erdal E and Atabey N: MiR-181a-5p is downregulated in hepatocellular carcinoma and suppresses motility, invasion and branching-morphogenesis by directly targeting c-Met. Biochem Biophys Res Commun 450: 1304-1312, 2014

40. Yang CH, Yue J, Pfeffer SR, Fan M, Paulus E, Hosni-Ahmed A, Sims M, Qayyum S, Davidoff AM, Handorf CR, et al: MicroRNA-21 promotes glioblastoma tumorigenesis by downregulating IGFBP3. J Biol Chem 289: 25079-25087, 2014.

41. Chen X, Wang J, Cheng L and Lu MP: miR-18a downregulates DICER1 and promotes proliferation and metastasis of nasopharyngeal carcinoma. Int J Clin Exp Med 7: 847-855, 2014.

42. Mo X, Zhang F, Liang H, Liu M, Li H and Xia H: miR-544a promotes the invasion of lung cancer cells by targeting cadherina 1 in vitro. Onco Targets Ther 7: 895-900, 2014.

43. Zang W, Wang Y, Du Y, Xuan X, Wang T, Li M, Ma Y, Li P, Chen X, Dong Z, et al: Differential expression profiling of microRNAs and their potential involvement in esophageal squamous cell carcinoma. Tumour Biol 35: 3295-3304, 2014.

44. Cheng W, Gao J, Zhang Z, Ge J, Xu F and Wei Z: Study on microRNAs in urothelial carcinoma (II grade) of the bladder. J Med Postgraduates China 23: 48-52, 2010.

45. Huang Z, Huang S, Wang Q, Liang L, Ni S, Wang L, Sheng W, He X and Du X: MicroRNA-95 promotes cell proliferation and targets sorting Nexin 1 in human colorectal carcinoma. Cancer Res 71: 2582-2589, 2011.

46. Yao T, Rao Q, Liu L, Zheng C, Xie Q, Liang J and Lin Z: Exploration of tumor-suppressive microRNAs silenced by DNA hypermethylation in cervical cancer. Virol J 10: 175, 2013.

47. Li WG, Yuan YZ, Qiao MM and Zhang YP: High dose glargine alters the expression profiles of microRNAs in pancreatic cancer cells. World J Gastroenterol 18: 2630-2639, 2012.

48. Xiao Z, Ching Chow S, Han Li C, Chun Tang S, Tsui SK, Lin Z and Chen Y: Role of microRNA-95 in the anticancer activity of Brucein D in hepatocellular carcinoma. Eur J Pharmacol 728: 141-150, 2014.

49. Huang X, Taeb S, Jahangiri S, Emmenegger U, Tran E, Bruce J, Mesci A, Korpela E, Vesprini D, Wong CS, et al: miRNA-95 mediates radioresistance in tumors by targeting the sphingolipid phosphatase SGPP1. Cancer Res 73: 6972-6986, 2013.

50. Chen X, Chen S, Hang W, Huang $\mathrm{H}$ and Ma H: MiR-95 induces proliferation and chemo- or radioresistance through directly targeting sorting nexin1 (SNX1) in non-small cell lung cancer. Biomed Pharmacother 68: 589-595, 2014.

51. Skalsky RL and Cullen BR: Reduced expression of brain-enriched microRNAs in glioblastomas permits targeted regulation of a cell death gene. PLoS One 6: e24248, 2011.

52. Wan J, Zhou J, Zhao H, Wang M, Wei Z, Gao H, Wang Y and Cui H: Sonic hedgehog pathway contributes to gastric cancer cell growth and proliferation. Biores Open Access 3: 53-59, 2014.

53. Huang L, Walter V, Hayes DN and Onaitis M: Hedgehog-GLI signaling inhibition suppresses tumor growth in squamous lung cancer. Clin Cancer Res 20: 1566-1575, 2014

54. An Y, Cai B, Chen J, Lv N, Yao J, Xue X, Tu M, Tang D, Wei J, Jiang K, et al: MAP3K10 promotes the proliferation and decreases the sensitivity of pancreatic cancer cells to gemcitabine by upregulating Gli-1 and Gli-2. Cancer Lett 329: 228-235, 2013. 
55. Luongo C, Ambrosio R, Salzano S, Dlugosz AA, Missero C and Dentice $\mathrm{M}$ : The sonic hedgehog-induced type 3 deiodinase facilitates tumorigenesis of basal cell carcinoma by reducing Gli2 inactivation. Endocrinology 155: 2077-2088, 2014.

56. Perrot CY, Gilbert C, Marsaud V, Postigo A, Javelaud D and Mauviel A: GLI2 cooperates with ZEB1 for transcriptional repression of $C D H 1$ expression in human melanoma cells. Pigment Cell Melanoma Res 26: 861-873, 2013.

57. Lattanzio R, Marchisio M, La Sorda R, Tinari N, Falasca M, Alberti S, Miscia S, Ercolani C, Di Benedetto A, Perracchio L, et al; CINBO (Consorzio Interuniversitario Nazionale per Bio-Oncologia): Overexpression of activated phospholipase $\mathrm{C} \gamma 1$ is a risk factor for distant metastases in T1-T2, N0 breast cancer patients undergoing adjuvant chemotherapy. Int J Cancer 132: 1022-1031, 2013.

58. Ma LW, Zhou ZT, He QB and Jiang WW: Phospholipase C- $\gamma 1$ expression correlated with cancer progression of potentially malignant oral lesions. J Oral Pathol Med 42: 47-52, 2013.

59. Behjati S, Tarpey PS, Sheldon H, Martincorena I, Van Loo P, Gundem G, Wedge DC, Ramakrishna M, Cooke SL, Pillay N, et al: Recurrent PTPRB and PLCG1 mutations in angiosarcoma. Nat Genet 46: 376-379, 2014

60. Vaqué JP, Gómez-López G, Monsálvez V, Varela I, Martínez N, Pérez C, Domínguez O, Graña O, Rodríguez-Peralto JL, Rodríguez-Pinilla SM, et al: PLCG1 mutations in cutaneous T-cell lymphomas. Blood 123: 2034-2043, 2014.

61. Meyer RD, Husain D and Rahimi N: c-Cbl inhibits angiogenesis and tumor growth by suppressing activation of PLC $\gamma 1$. Oncogene 30: 2198-2206, 2011.
62. Phillips-Mason PJ, Kaur H, Burden-Gulley SM, Craig SE and Brady-Kalnay SM: Identification of phospholipase $\mathrm{C}$ gamma1 as a protein tyrosine phosphatase mu substrate that regulates cell migration. J Cell Biochem 112: 39-48, 2011.

63. Yang J, Song X, Chen Y, Lu XA, Fu Y and Luo Y: PLC $\gamma 1-P K C \gamma$ signaling-mediated Hsp90 $\alpha$ plasma membrane translocation facilitates tumor metastasis. Traffic 15: 861-878, 2014.

64. Choi AY, Choi JH, Hwang KY, Jeong YJ, Choe W, Yoon KS, Ha J, Kim SS, Youn JH, Yeo EJ, et al: Licochalcone A induces apoptosis through endoplasmic reticulum stress via a phospholipase $\mathrm{C} \gamma 1-, \mathrm{Ca}^{2+}$, and reactive oxygen species-dependent pathway in HepG2 human hepatocellular carcinoma cells. Apoptosis 19: 682-697, 2014

65. Kortum RL, Rouquette-Jazdanian AK, Miyaji M, Merrill RK, Markegard E, Pinski JM, Wesselink A, Nath NN, Alexander CP, Li W, et al: A phospholipase C- $\gamma 1$-independent, RasGRP1-ERK-dependent pathway drives lymphoproliferative disease in linker for activation of T cells-Y136F mutant mice. J Immunol 190: 147-158, 2013.

66. Zhang Q, Yu C, Peng S, Xu H, Wright E, Zhang X, Huo X, Cheng E, Pham TH, Asanuma K, et al: Autocrine VEGF signaling promotes proliferation of neoplastic Barrett's epithelial cells through a PLC-dependent pathway. Gastroenterology 146: 461-472.e6, 2014.

67. Yang JM, Vassil AD and Hait WN: Activation of phospholipase C induces the expression of the multidrug resistance $(M D R I)$ gene through the Raf-MAPK pathway. Mol Pharmacol 60: 674-680, 2001. 